\title{
Hard Two-Photon Contribution to Elastic Lepton-Proton Scattering Determined by the OLYMPUS Experiment
}

\author{
Dmitry Khaneft ${ }^{* \dagger}$ \\ Helmholtz-Institut Mainz \\ E-mail: khaneftd@kph.uni-mainz.de
}

\begin{abstract}
The OLYMPUS collaboration has recently performed a precise measurement of the positronproton to electron-proton elastic scattering cross section ratio, $R_{2 \gamma}$, over a wide range of the virtual photon polarization, $0.456<\epsilon<0.978$. This provides a direct measure of hard two-photon exchange in elastic lepton-proton scattering widely thought to explain the discrepancy observed between polarized and unpolarized measurements of the proton form factor ratio, $\mu_{p} G_{E}^{p} / G_{M}^{p}$. The OLYMPUS results are significantly lower than theoretical calculations that explain the observed discrepancy in terms of two-photon exchange but are in good agreement with predictions based on phenomenological fits to the available form factor data.

The motivation for measuring hard two-photon exchange is presented followed by a description of the OLYMPUS experiment and analysis. The important role of soft two-photon contributions from radiative corrections for the analysis is highlighted. Then, the OLYMPUS results are presented and compared with various theoretical calculations. Finally, a comparison between the OLYMPUS and two analogous modern experiments with one of the latest theoretical model is shown.
\end{abstract}

XXV International Workshop on Deep-Inelastic Scattering and Related Subjects

3-7 April 2017

University of Birmingham, $U K$

\footnotetext{
* Speaker.

${ }^{\dagger}$ for the OLYMPUS Collaboration.
} 


\section{Introduction}

The proton's electromagnetic form factors, $G_{E}\left(Q^{2}\right)$ and $G_{M}\left(Q^{2}\right)$, have been measured over the past several decades. First experiments measured unpolarized elastic electron-proton scattering cross sections at different scattering angles and momentum transfer $Q^{2}$. The proton form factor ratio was extracted using the Rosenbluth separation method [1-7]. As shown in Fig. 1, results are mostly consistent with unity across the whole $Q^{2}$ range. With an advent of polarization technologies, a new type of experiment became possible. The same ratio was determined by scattering polarized electron beams off unpolarized protons and measuring the polarization of recoil protons. New results [8-14] came as a big surprise, the ratio extracted using the polarization technique had a clear $Q^{2}$ dependence as shown in Fig. 1. After the discrepancy was discovered, both types of experiments were repeated in order to eliminate possible systematic errors in old measurements; no solution have been found. Therefore, there must be some other unknown effects at play.

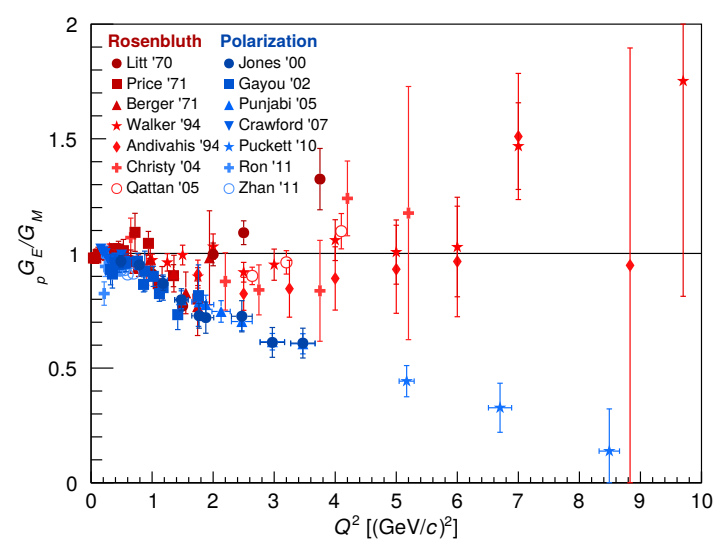

Figure 1: The proton's form factor ratio extracted using Rosenbluth separation (red) and polarization transfer data (blue).

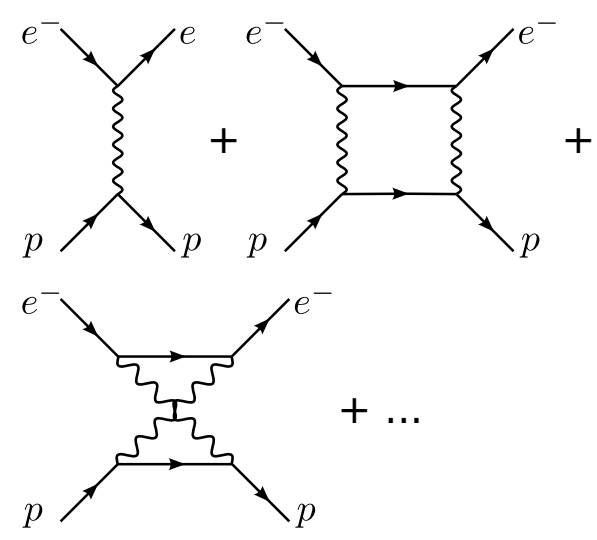

Figure 2: Examples of one- and some twophoton exchange Feynman diagrams contributing to the elastic electron-proton and positronproton scattering.

Many theoretical attempts have been made to explain the difference and to reconcile two sets of data. The previously unaccounted hard two-photon exchange (see Fig. 2) contribution was considered to be the cause of the discrepancy. The two-photon exchange contribution affects stronger the Rosenbluth method in comparison to the polarization technique [15].

Experimentally, the size of two-photon exchange can be directly determined by measuring the elastic scattering cross section ratio $\sigma_{e^{+} p} / \sigma_{e^{-}}$. In the one-photon exchange approximation, the cross section ratio $\sigma_{e^{-}} / \sigma_{e^{+}}$is equal to unity as there is no asymmetry between electrons and positrons. On the other hand, the interference term between one- $\mathscr{M}_{1 \gamma}$ and two-photon $\mathscr{M}_{2 \gamma}$ exchange amplitudes has an opposite sign for electrons and positrons. The cross section ratio, in terms of these amplitudes, can be written at the leading order in $\alpha$ as

$$
\frac{\sigma_{e^{+} p}}{\sigma_{e^{-}} p}=\frac{\left|\mathscr{M}_{1 \gamma}\right|^{2}+2 \mathscr{R}\left(\mathscr{M}_{1 \gamma} \mathscr{M}_{2 \gamma}\right)}{\left|\mathscr{M}_{1 \gamma}\right|^{2}-2 \mathscr{R}\left(\mathscr{M}_{1 \gamma} \mathscr{M}_{2 \gamma}\right)}
$$


The lack of precise data and the fact that all theoretical calculations are model dependent motivated the OLYMPUS collaboration to perform an accurate measurement of two-photon exchange contribution.

\section{Experiment}

At the core of the OLYMPUS experiment was an internal hydrogen gas target. It was surrounded by an eight-coil toroidal magnet and detectors were located in two horizontal sectors on both sides of the beamline (see Fig. 5). Each sector had a drift chamber and a set of time-offlight scintillator bars. The former were used for tracking and particle identification while the latter served as a main trigger for the data acquisition system as well as for particle identification. Three independent systems were used for luminosity determination: 1) The $12^{\circ}$ degree monitors were built from multi-wire proportional chambers and gas electron multipliers and detected leptonproton elastic scattering; 2) The Symmetric Møller/Bhabha luminosity monitor consisted of two identical Cherenkov calorimeters that were placed symmetrically in each sector at $1.29^{\circ}$, it used Møller and Bhabha scattering; 3) Additionally, the luminosity was estimated using the slow control system data: the beam current, target density, and measurement time.

The data was collected at the DORIS storage ring at DESY, in Hamburg, Germany. The facility provided $2 \mathrm{GeV}$ electron and positron beams which were normally changed every 24 hours. In total, $4.5 \mathrm{fb}^{-1}$ of data were collected during two data taking periods.

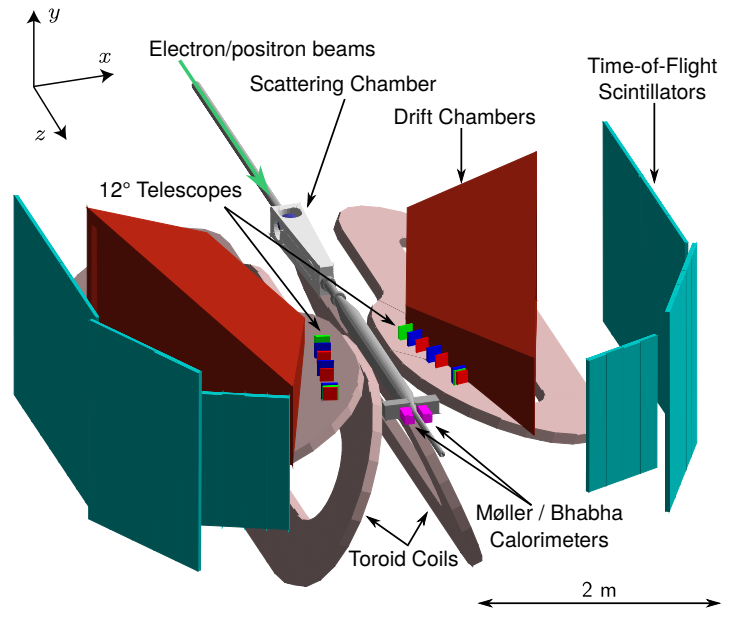

Figure 3: A solid-model representation, with the top four magnet coils hidden, of the OLYMPUS detector with the top four magnet coils removed to show the instrumented horizontal sectors.

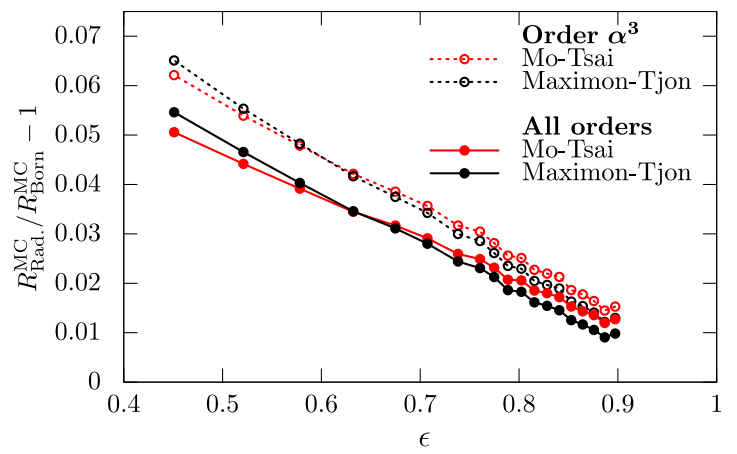

Figure 4: Size of two-photon exchange corrections according to Mo-Tsai calculations.

\section{Results}

Due to very complicated nature of the OLYMPUS experiment, the data analysis heavily relied on a full Monte Carlo simulation. For this purpose, a radiative $e^{+} p$ and $e^{-} p$ event generator was 
developed to account for the soft two-photon exchange, vertex corrections, vacuum polarizations, and contributions from bremsstrahlung. Figure 4 illustrates approximate size of radiative corrections to OLYMPUS relative to the born approximation. It allowed to simulate not only radiative effects but also include those coming from different beam species, beam position and slope, track reconstruction efficiency, etc. The elastic positron-proton to electron-proton scattering cross section ratio was estimated by comparing number of events in the data and Monte Carlo simulation as follows:

$$
R_{2 \gamma}=\frac{\sigma_{e^{+} p}}{\sigma_{e^{-} p}}=\frac{N_{e^{+} p}^{\text {Data }}}{N_{e^{-} p}^{\text {Data }}} \times \frac{N_{e^{-} p}^{M C}}{N_{e^{+} p}^{M C}},
$$

where $N_{i}$ are luminosity normalized experimental and Monte Carlo counts.

The OLYMPUS results [16] are shown in Fig. 5 together with a number of theoretical calculations. As can bee seen, the measured two-photon exchange contribution is very small and consistent with unity at $\epsilon>0.65$. The only significantly large deviation from unity is seen at $\epsilon=0.46$ where $R_{2 \gamma}$ goes slightly above $2 \%$. Recent dispersion calculations of Blunden [17] seem to overestimate the size of two-photon exchange while capturing its general shape. At the same time, phenomenological predictions of Bernauer and dispersion calculations Tomalak consistently match the data.

Figure 6 shows a difference between OLYMPUS, two other modern experiments CLAS [18] and VEPP-3 [19], and the newest calculations of Blunden. Theoretical values were calculated for each data point individually taking experimental $\epsilon$ and $Q^{2}$ into account. All three experiments are comparable to each other and show smaller measured values of two-photon exchange than Blunden's calculations predict across the whole $\epsilon$ range.

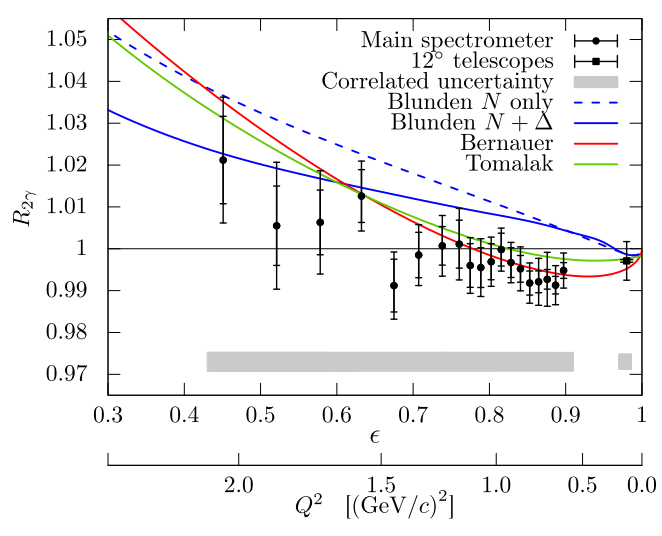

Figure 5: OLYMPUS result for two-photon effect using the Mo-Tsai [20] prescription for radiative corrections to all orders. Uncertainties shown are statistical (inner bars), uncorrelated systematic (added in quadrature, outer bars), and correlated systematic (gray band). Note the $12^{\circ}$ data point at $\epsilon=0.978$ is completely dominated by systematic uncertainties.

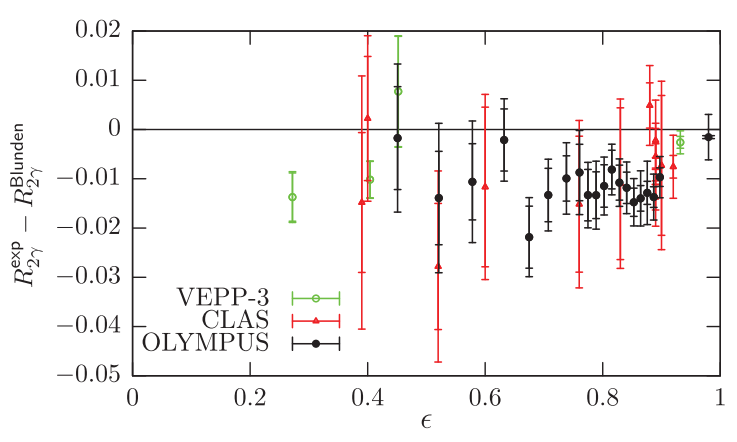

Figure 6: Comparison of the recent results to the calculation by Blunden. The data are in good agreement, but generally fall below the prediction. Please note that data at similar $\epsilon$ values have been measured at different $Q^{2}$. Also note that the VEPP-3 data have been normalized to the calculation at high $\epsilon$. 


\section{Conclusions}

It has been shown that in the kinematic range of the OLYMPUS experiment the two-photon exchange contribution to the elastic electron-proton to positron-proton scattering cross section ratio is on the level of $0 \%$ to $1 \%$ depending on the $\epsilon$. Thus, it does not allow to explain the existing discrepancy between Rosenbluth separation method and polarization technique. On the other hand, two-photon exchange shows a correlation with $Q^{2}$, therefore, it is possible that at higher energies its effect will be significant enough to reconcile the two types of measurement. In order to give a definitive answer, new theoretical models, which include more intermediate states, are needed as well as new experiments in the high energy range.

\section{References}

[1] J. Litt et al. Physics Letters B, 31(1):40 - 44, 1970.

[2] L. E. Price et al. Phys. Rev. D, 4:45-53, Jul 1971.

[3] Ch. Berger et al. Physics Letters B, 35(1):87 - 89, 1971.

[4] R. C. Walker et al. Phys. Rev. D, 49:5671-5689, Jun 1994.

[5] L. Andivahis et al. Phys. Rev. D, 50:5491-5517, Nov 1994.

[6] M. E. Christy et al. Phys. Rev. C, 70:015206, Jul 2004.

[7] I. A. Qattan et al. Phys. Rev. Lett., 94:142301, Apr 2005.

[8] M. K. Jones et al. Phys. Rev. Lett., 84:1398-1402, Feb 2000.

[9] O. Gayou et al. Phys. Rev. Lett., 88:092301, Feb 2002.

[10] V. Punjabi et al. Phys. Rev. C, 71:055202, May 2005.

[11] C. B. Crawford et al. Phys. Rev. Lett., 98:052301, Jan 2007.

[12] A. J. R. Puckett et al. Phys. Rev. Lett., 104:242301, Jun 2010.

[13] G. Ron et al. Phys. Rev. C, 84:055204, Nov 2011.

[14] X. Zhan et al. Physics Letters B, 705(1-2):59-64, 2011.

[15] A. V. Afanasev et al. Phys. Rev. D, 72:013008, Jul 2005.

[16] B. S. Henderson et al. Phys. Rev. Lett., 118:092501, Mar 2017.

[17] P. G. Blunden and W. Melnitchouk. Phys. Rev. C, 95:065209, Jun 2017.

[18] D. Rimal et al. 2016. arXiv:1603.00315 [nucl-ex].

[19] I. A. Rachek et al. Phys. Rev. Lett., 114:062005, Feb 2015.

[20] L. W. Mo and Y. S. Tsai. Rev. Mod. Phys., 41:205-235, Jan 1969. 